\title{
Loss induced multiple symmetry breakings in the Fermi Pasta Ulam recurrence process
}

\author{
Guillaume Vanderhaegen ${ }^{1}$, Pascal Szriftgiser ${ }^{1}$, Matteo Conforti ${ }^{1}$, Alexandre Kudlinski ${ }^{1}$, Stefano Trillo $^{2}$, \\ Arnaud Mussot ${ }^{1}$ \\ 1. University of Lille, CNRS, UMR 8523 - PhLAM - Physique des Lasers Atomes et Molécules, F-59000 Lille, France \\ 2. Department of Engineering, University of Ferrara, 44122 Ferrara, Italy
}

Fermi Pasta Ulam (FPU) recurrence process describes the ability of a nonlinear system to excite multiple modes and then to return to its initial state. In fiber optics, such process has been investigated within the framework of modulation instability (MI) [1], corresponding to growth of weak perturbations at the expense of a strong pump. Nonlinear stage of MI and FPU recurrences in optical fibers have been widely investigated and many results have been obtained with an efficient compensation of the losses [2]. Investigations on water waves [3] demonstrated that dissipation of their water tank forces the system to operate in a single regime. Here, we provide a deeper and complete study on the impact of dissipation of the FPU dynamics. Thanks to a fiber optics system, we have been able to control the loss coefficient, allowing us to trigger the different corresponding FPU regimes.

Using a setup similar to the one in [2] and based on a multiple HOTDR system, we managed to record FPU recurrences in power and phase along the fiber length as a function of the loss, Fig. 1. The dissipation is controlled thanks to a counter-propagating Raman pump whose power is tuned to get an effective loss coefficient. A direct relation between the Raman pump power and effective loss coefficient is obtained, allowing a direct comparison between experiments (left panel) and simulations (right panel). Three different regions are discernible. In the $3^{r d}$
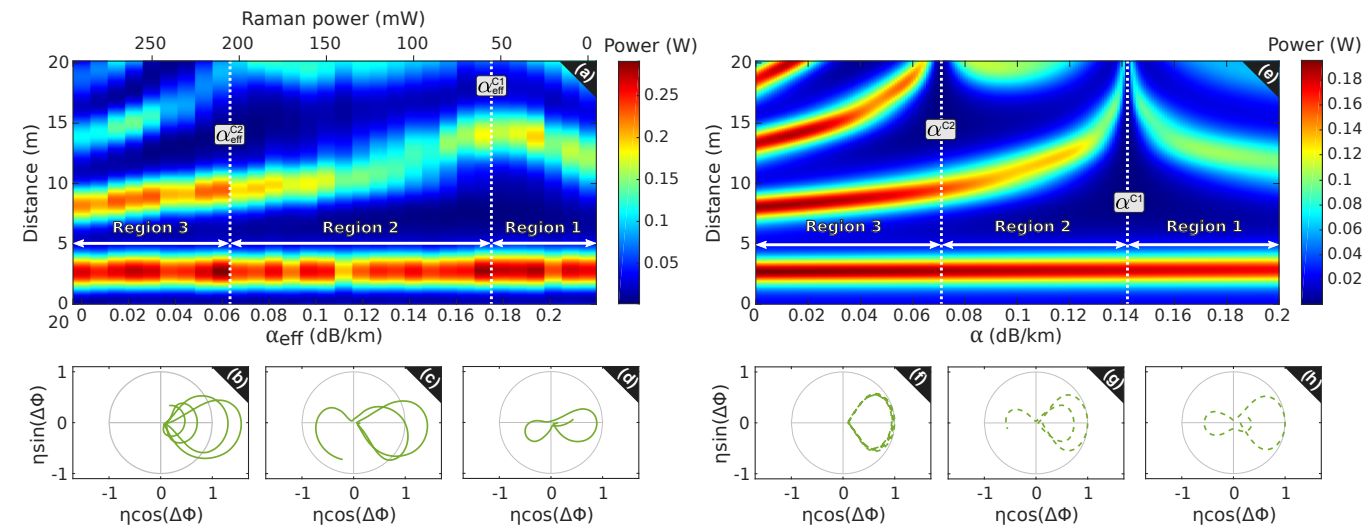

Figure 1: 2D-plot of the signal power evolution along the fiber length as a function of loss (a) in experiments and (e) in numerics. An example of phase-plane evolution is plotted for each region; (b)-(d) for experiments, and (f)-(h) for numerics.(b) and (f) $\alpha=0.007 \mathrm{~dB} / \mathrm{km}$, (c) and (g) $\alpha=0.09 \mathrm{~dB} / \mathrm{km}$, (d) and (h) $\alpha=0.2 \mathrm{~dB} / \mathrm{km}$.

one (quasi-perfect compensation of the losses), we observe 4 recurrences (Fig. 1. (a)), corresponding to 4 loops located in the right-half side of the phase plane (Fig. 1. (b)). This means that the maximum compression points remain at the same temporal location at each recurrence [2]. In the $2^{\text {nd }}$ region (partial compensation), the first two recurrences are in phase and the $3^{\text {rd }}$ one is shifted, as shown in the phase plane (Fig. 1. (c), 2 loops in the semi-right side and one in the left one). In the $1^{s t}$ region (the loss is close to the fiber intrinsic value), there are 2 recurrences characterized by one loop in each semi-plane of the phase plane (Fig. 1. (d)). This means that these recurrences are phase-shifted [2]. Each region is separated by a critical value $\alpha^{C N}$, determined from phase evolution (not shown here for clarity). Experiments are in good agreement with numerics (compare Fig. 1. (a)-(d) to (e)-(h)).

To conclude, we reported a complete study on dissipation impact on FPU dynamics in optical fibers thanks to the accurate control of the losses and to our non-destructive distributed measurement setup [2]. It reveals that dissipation strongly impacts the dynamics of FPU recurrence by forcing the system to evolve into several specific regimes.

\section{References}

[1] G. Van Simaeys, Ph. Emplit, and M. Haelterman, "Experimental Demonstration of the Fermi-Pasta-Ulam Recurrence in a Modulationally Unstable Optical Wave," Physical Review Letters 87, 033902 (2001).

[2] A. Mussot, C. Naveau, M. Conforti, A. Kudlinski, F. Copie, P. Szriftgiser, and S. Trillo, 'Fibre multi-wave mixing combs reveal the broken symmetry of Fermi-Pasta-Ulam recurrence," Nature Photonics 12, 303-308 (2018).

[3] O. Kimmoun, H. C. Hsu, H. Branger, M. S. Li, Y. Y. Chen, C. Kharif, M. Onorato, E. J. R. Kelleher, B. Kibler, N. Akhmediev, and A. Chabchoub, ”Modulation Instability and Phase-Shifted Fermi-Pasta-Ulam Recurrence," Scientific Reports 6, 28516 (2016). 